Bull. Mater. Sci. vol. 19. Vo. 2. April 1996, pp. 417. 422, , Printed in India.

\title{
Detection of surface lattice defects using line scan method ${ }^{\dagger}$
}

\author{
M V H RAO*. B K MATHUR and K L CHOPRA \\ Department of Physics and Metcorology, Indian Institute of Technology, Kharagpur 721302. \\ India \\ * Present address: CMVT, Kushaiguda, Hyderabad 500762. India
}

\begin{abstract}
The presence of different kinds of surface lattice defects such as missing atom. interstitial atom. line defects, in graphite single erystal have been identified by using scanning tunneling microscope. These defects cause displacement of atoms from their mean position and lattice strain is introduced. By measuring the displacement of atoms from their mean position. lattice strain has been culculated. It is found that among single point defects, vatancies cause maximum lattice strain.
\end{abstract}

Keywords. STM: lattice defects: graphite single crystal.

\section{Introduction}

Since the advent of STM by Binnig ot al (1982). graphite has been the most widely studied material for achicving atomic resolution. First topographical images of graphite were recorded by Binnig et al (1986) under UHV conditions. The capability of STM in achieving atomic resolution in air was demonstrated by Park and Quate (1986). Bryant et al (1986) reported resolution better than $2 \AA$. Tomanek et al (1987) reported a strong asymmetry in the tunneling current between neighbouring carbon atoms in the hexagonal ring and explained it in terms of a purely electronic effect, arising from the symmetry of the states scanned by STM. which dominates over the topography of graphite. Rao et al (1992) used a line scan technique to identify electronically different surface atoms.

The presence of surface defects such as interstitial atoms, missing atoms, line defects can be casily identified by the line scan method (Rao et al 1992). These defects are associated with displacement of atoms from their mean positions and the measurement of these atomic displacements give us a quantitative estimation of the surface strain induced by these defects. STM surface topography combined with the line scan technique can be very useful in identifying and analyzing these defects. Coratger ot al (1990) studied defects induced by carbon bombardment on HOPG surface. They observed ridge structures running along $[1 / 100)]$ direction and point defects which are identified by smearing up of ( $\mathrm{site}$ by clcctron cloud from neighbouring atoms thereby producing flat current response instead of a minmum During our investigations, STM images of some non-uniform regions of graphite surface are taken to analyze crystallographic surface defects present on it

\section{Experimental}

A single crystal of highly oriented pyrolitic graphic (HOPG) with the $(0001$ ) surface facing the tip is mounted on a nickel stub with silver paint providing the electrical 


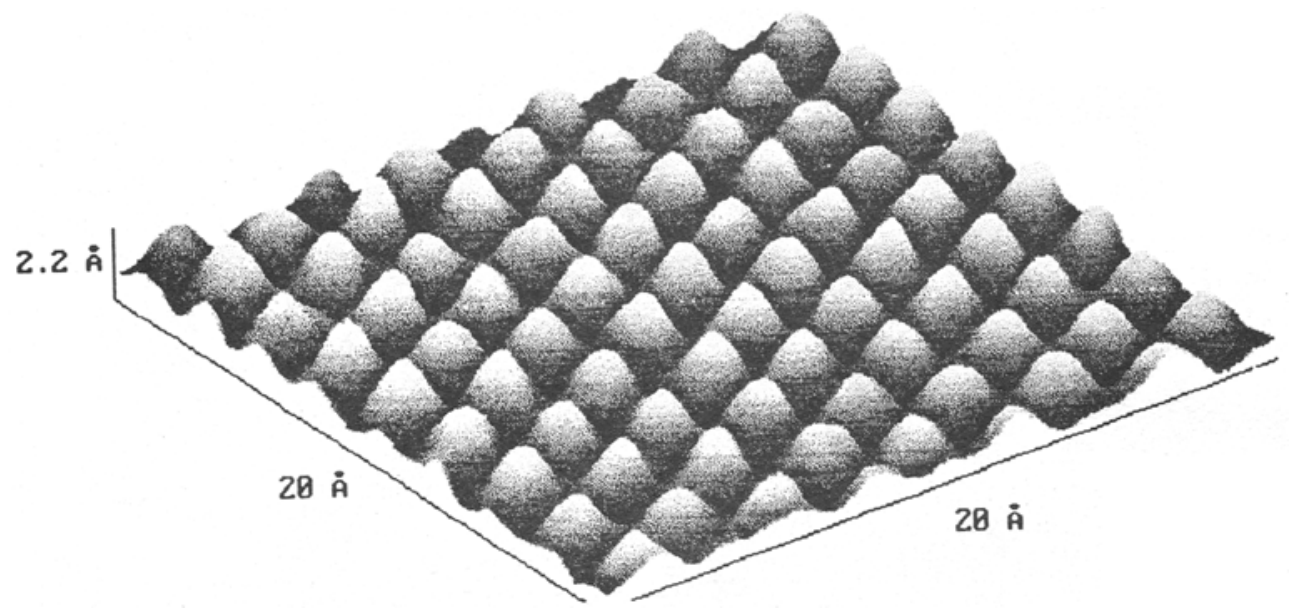

Figure 1. 3-D STM image of (0001) surface of freshly cleaved HOPG recorded in air, at a tunneling current of $1 \mathrm{nA}$ and bias voltage of $0.1 \mathrm{~V}$. Interatomic distance is $2.46 \AA$. All the prominent peaks in the image represent $A$ kind of atoms.

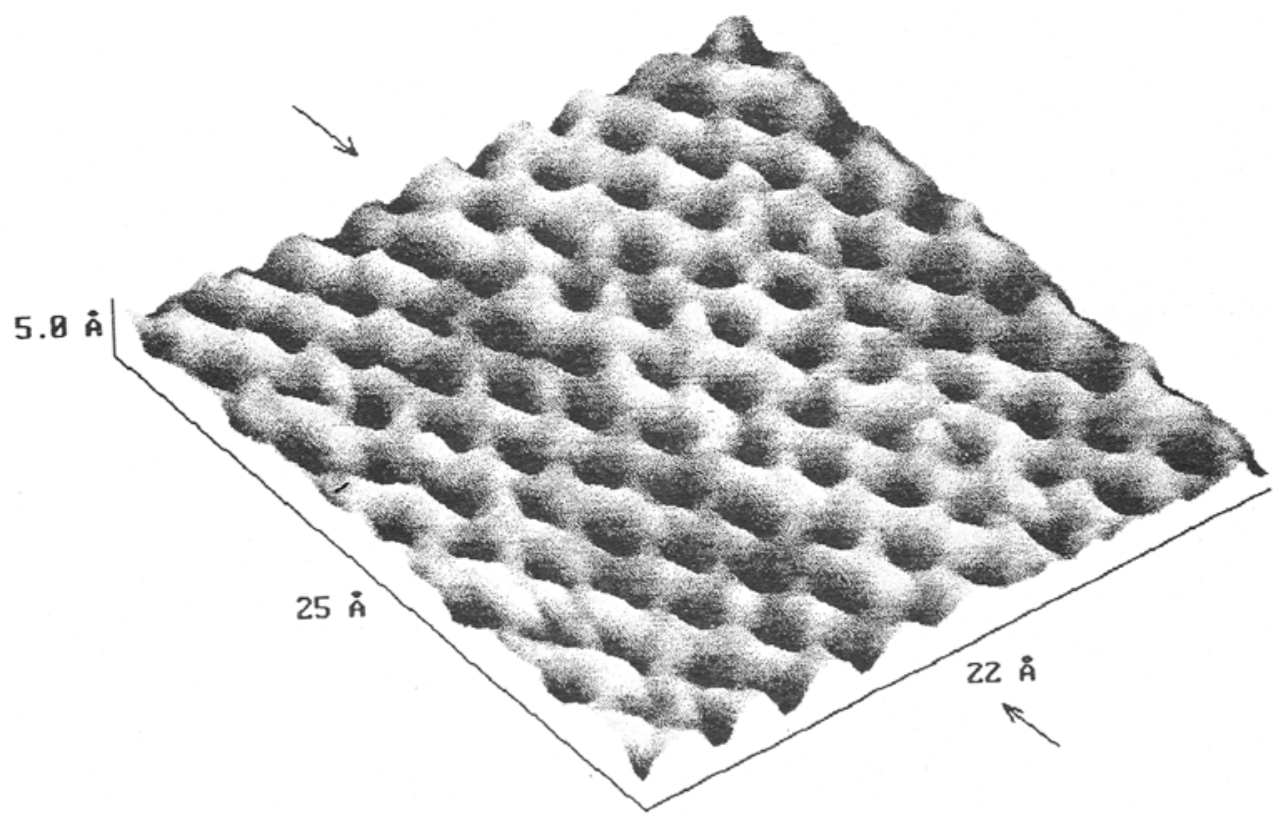

Figure 2. 3-D STM image of a monolayer step on the graphite surface.

contact. AS HOPG crystal is chemically inert to atmospheric exposure, the same sample surface can be used repeatedly for either monitoring the tip quality or for recalibration of STM. If the surface is damaged due to repeated handling, then a fresh surface can be easily obtained either by cleaving with a sharp razor blade or by gently removing the top layer by sticking it to a cellophane tape. 
A bias voltage of $0.15 \mathrm{~V}$ was applied to the sample and it was brought into tunneling range with a set value of $1 \mathrm{nA}$. Initially large areas are scanned to locate atomically smooth regions on the surface. The scan range is gradually reduced and at each range the surface is rescanned until the required resolution is achieved. If necessary the sample is moved under the tip to bring the planar regions under the scan preview by using the $\mathrm{X}, \mathrm{Y}$ offset piezos. Several scans with atomic resolution extending over an area up to a thousand square angstroms were recorded with high reproducibility (figure 1).
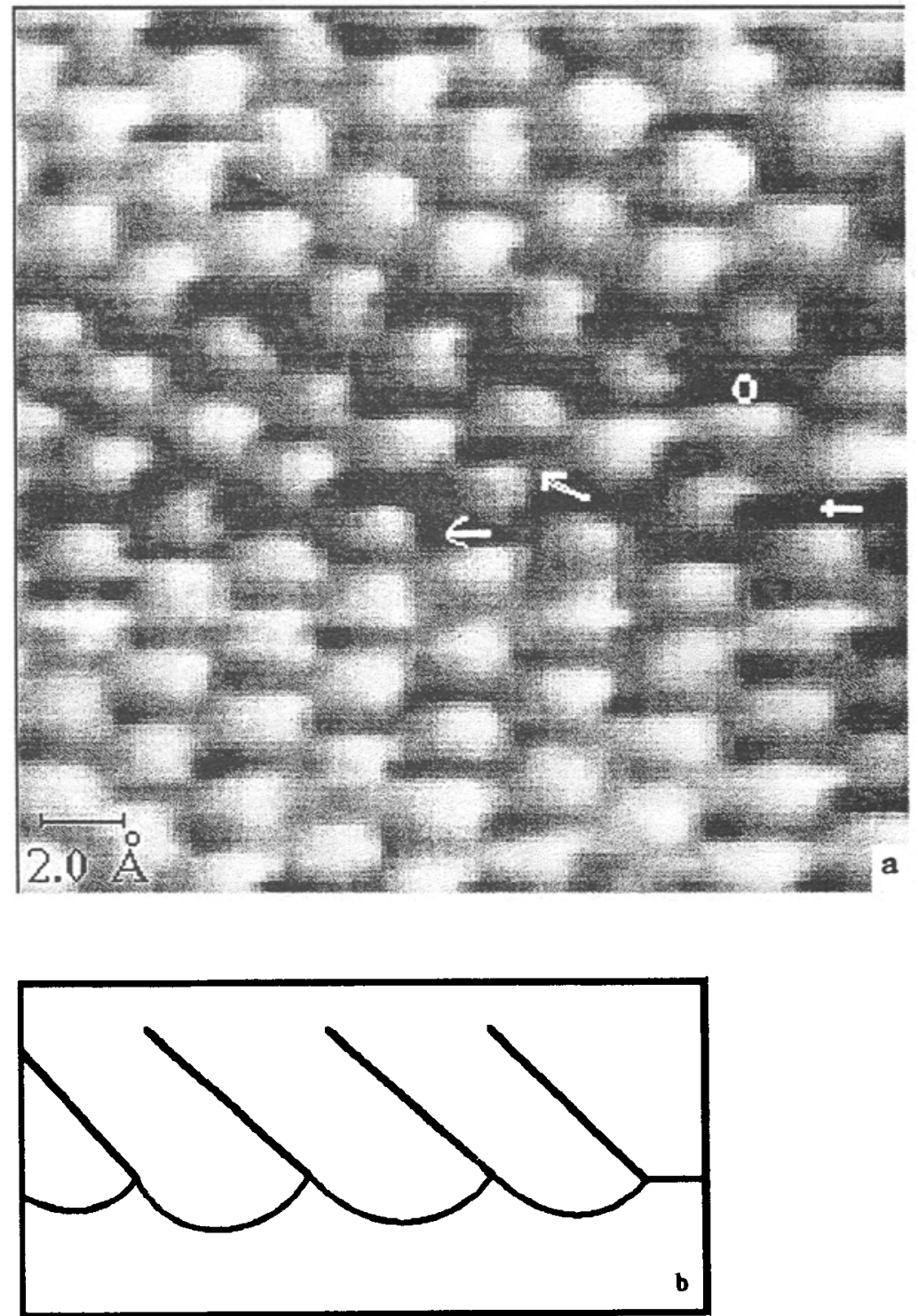

Figure 3. (a) STM image of a line defect on graphite (0001) surface. Scan area is $20 \AA \times 20 \AA$ and (b) nature of propagation of line defect in (a). 


\section{Results and discussions}

The surface of the graphite single crystal (0001 plane) is not only atomically flat over certain areas extending up to a thousand square angstroms but also a treasure house of different kinds of surface lattice defects. It is during the surface topographic studies that these defects are detected and identified.

Figure 2 is a topographic image of the surface where the right side of the image is brighter (higher level) than the left side. The boundary is marked with arrows in the figure. Also the height variation in the topograph is $5 \AA$ as compared with a height variation of $2 \cdot 2 \AA$ for a perfectly defect free surface in figure 1 . This indicates that the line running along the marked arrows is a mono-atomic step where the top layer has ended. This is also confirmed by the fact that the height variations within the right and left hand side of the step are within $2 \cdot 2 \AA$. The individual bright spot on the left side of the arrows (bottom layer) are likely to be individual carbon atoms as their peak to valley height change is around $5 \AA$, which is the same as that of a monolayer step. Figure $3 a$ shows the presence of a line defect (marked by arrows), leaving a trail of vacancy point defects. The defect zone is about $10 \AA$ wide running through the middle of the image. The nature of propagation of line defect is of the type shown in figure $3 \mathrm{~b}$.

A defect filled area of the surface is shown in figure 4 which contains several point defects. In this topograph we can identify vacant sites and displaced atoms by drawing

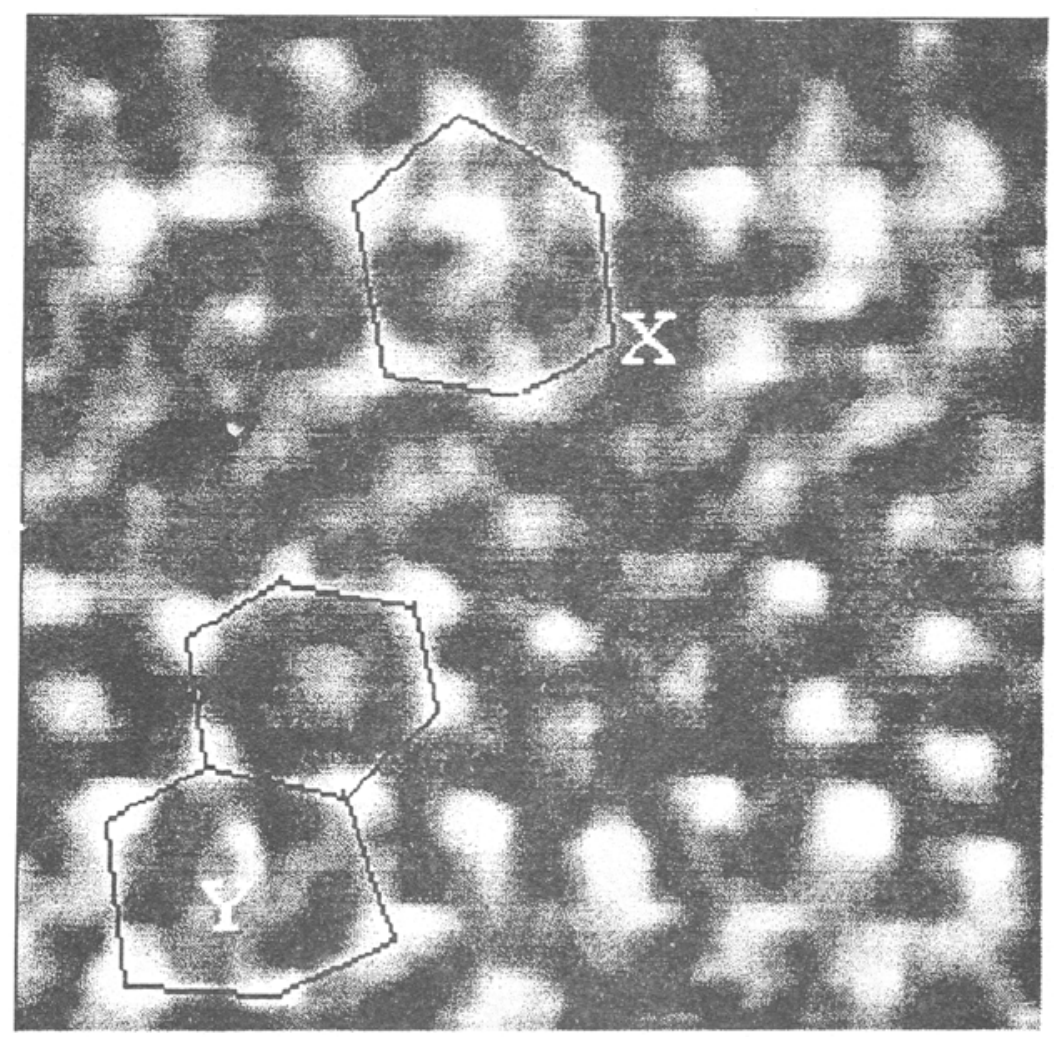

Figure 4. Image showing a large number of point defects. 


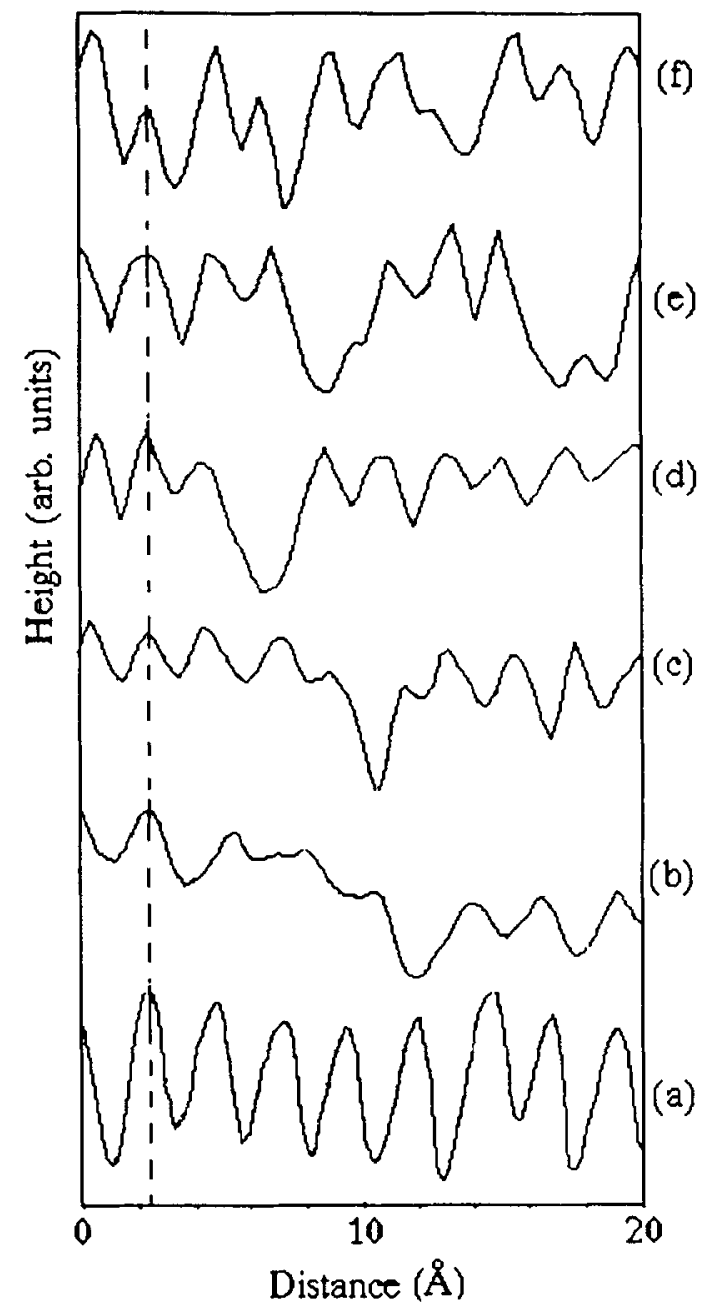

Figure 5. Line profles taken along the [1100] direction (i.e. only A kind of atoms) at different locations on graphite $(0001)$ surface indicating the presence of surface defects. For comparison purpose a line profile on a perfect crystal is presented at the bottom.

a hexagonal unit cell and identifying an atom at each corner and one at the centre of the unit cell. We can clearly see in the figure that an A type atom is missing at position $X$. The atom at site $Y$ is displaced from its mean position (which is at the centre of the unit celi). This displacement has generated a strain in the lattice as is evidenced by the distorted hexagonal ring around $\mathrm{Y}$.

Using the line scan procedure, atomic corrugation profiles are recorded across the defect sites (figure 5). As revealed from topographic data, the ex tent of strain is confined to within three atomic distances from the site of the defect, a length of $20 \AA$ is chosen to compare the atomic displacements and strain produced due to different types of surface defects. Line profiles have been taken in the [1100] direction to identify point defects for A type of atoms. Some of these line profiles are shown in figure 5 . For comparison purpose a line profile taken along defect free [1100] direction is shown in $a$. The line 
Table 1. Average fractional displacement (strain) of atoms from their mean position.

\begin{tabular}{lccc}
\hline & \multicolumn{3}{c}{ Fractional displacement of atoms } \\
\cline { 2 - 4 } Kind of defect & $\begin{array}{c}\text { First } \\
\text { neighbour }\end{array}$ & $\begin{array}{c}\text { Second } \\
\text { neighbour }\end{array}$ & $\begin{array}{c}\text { Third } \\
\text { neighbour }\end{array}$ \\
\hline Vacancy & 0.43 & 0.37 & 0.21 \\
Frankel & 0.12 & 0.09 & 0.10 \\
Step & 0.23 & 0.11 & 0.05 \\
\hline
\end{tabular}

profile $b$ taken across a monolayer step gives us a step width of $\sim 5 \AA$. It is seen that an atomic row (at location 5) is missing at the boundary of the monolayer. Profile $c$ identifies the absence of an A type atom at location 4, while in $d$ the atom at site 3 is displaced to the right, thereby creating a Frankel type (vacancy + interstitial) of defect. Profile $e$ shows two vacancies within a distance of $20 \AA$ at locations 4 and 8 . The peaks appearing inside the vacant site troughs are likely to be due to the atoms in the layer just below the top layer. The profile $f$ taken in [1000] direction, where atomic sites $A$ and $B$ are identified, clearly shows the missing of a B type of atom and a displacement of next A site to the left.

By measuring the displacement of atoms around a defect, the strain induced by them on the neighbouring atoms has been calculated. The strain data given in table 1 corresponds to strain introduced by single identifiable surface defect. In order to determine rms strain and associated surface strain energy, it is necessary to consider the cumulative effect of all the lattice defects of different kinds, single and complex. Nevertheless we can conclude that for the case of simple defects, maximum lattice strain is caused by vacancies. Strain due to Frankel type of defect is less but propagates up to many atomic distances. Steps and terraces introduce short range strain.

\section{Conclusions}

It has been shown that by line scan technique, different types of surface lattice defects can be identified. By measuring the displacement of atoms from their mean position, lattice strain introduced by these defects can be estimated.

\section{References}

Binnig G, Rohrer H, Gerber Ch and Weibel E 1982 Phys. Rev. Lett. 4957

Binnig G, Fuchs H, Gerber Ch, Stoll E and Tosatti E 1986 Europhys. Lett. 131

Bryant A. Smith D P E and Quate C F 1986 Appl. Phys. Lett. 48832

Coratger R, Claverie A, Ajustron F and Beauvillain J 1990 Surf. Sci. 2277

Park Sang-Il and Quate C F 1986 Appl. Phys. Lett. 48112

Rao M V H, Mathur B K and Chopra K L 1992 J. Elect. Spectrosc. and Related Phenomena 6057

Tomanek D, Louie S G, Mamin H J, Abraham D W, Thomson R E, Ganz E and Clarke J 1987 Phys. Rev. B35 7790 